\title{
Celiac Disease and Liver Abnormalities: What Relationships?
}

\author{
D Tagzout* and A Tebaibia \\ Algiers Faculty of Medecine, Algiers University, El-Biar Hospital, Algeria
}

*Corresponding author: D Tagzout, Algiers Faculty of Medecine, Algiers University, El-Biar hospital, Algiers, Algeria.

Received Date: February 04, 2021

Published Date: February 22, 2021

\begin{abstract}
Introduction
Celiac disease (CD) may be associated with liver abnormalities (LA) and these abnormalities are often liver blood tests abnormalities corresponding to cryptogenic hypertransaminasemia or celiac hepatitis $(\mathrm{CH})$ which is reversible after strict gluten free diet (GFD). $\mathrm{CH}$ may be the only manifestation of unrecognized CD. Other LA may be associated, especially those of dysimmune origin or overload. The mechanisms of these associations are unclear and the role of the GFD is uncertain.
\end{abstract}

Keywords: Celiac disease; Hypertransaminasemia; Dysimmune liver disorders; Cirrhosis

\section{Introduction}

CD is an autoimmune enteropathy secondary to gluten ingestion in genetically predisposed individuals. Although its main target organ is the small intestine, CD is currently considered as a multisystemic disease, where the injury may affect different organs, particularly the liver. The GFD strict adherence, is so far the only effective treatment whose role on liver damage seems positive. A number of studies have shown that LA occurs at a higher frequency in patients with $\mathrm{CD}$, and these are often non-specific abnormalities of liver bioassays corresponding to $\mathrm{CH}$. Specific chronic LA may associate with $\mathrm{CD}$. A cautious diagnostic approach will not ignore the different associations.

\section{Celiac Hepatitis}

$\mathrm{CH}$ is the most common LA during $\mathrm{CD}$, observed in 13 to $60 \%$ of cases of untreated CD, with reversibility in more than 2 thirds of cases after 6 to 12 months of strict GFD [1]. This response to the GFD confirms this diagnosis. $\mathrm{CH}$ is asymptomatic and may be the only sign of previously unrecognized $\mathrm{CD}$. The elevation of transaminase levels is moderate, between 3 and 5 times the upper limit of the normal rate, and it has been shown that ALAT elevation in celiac children is correlated with the severity of villous atrophy and poor growth [2]. The hypothesis explaining this abnomality is an intestinal hyperpermeability with a massive passage of toxins and intra-luminal antigens through the portal circulation, and it has been shown that the intestinal permeability as well as the level of transaminases are corrected by a GFD [3].

\section{Celiac Disease and Liver Abnormalities}

Primary biliary cholangitis (PBC) and autoimmune hepatitis (AIH) may be associated with CD. The frequency of CD during PBC ranges from $1 \%$ to $7 \%$ and the frequency of $\mathrm{PBC}$ during $\mathrm{CD}$ varies from $0.1 \%$ to $3 \%$ depending on the studies [4]. CD is present in 4 to $6 \%$ of patients with type 1 or type 2 AIH. Cases of primary sclerosing cholangitis associated with $\mathrm{CD}$ have been described in the literature. The mechanism of these associations remains unknown but it may be a genetic susceptibility to immune disorders involving several 
antigenic targets (enterocytes, cholangiocytes, hepatocytes). The effect of GFD seems very weak without significant improvement in liver signs.

\section{Celiac Disease and Non Alcoholique Fatty Liver Disease}

$\mathrm{CD}$ is seen in 3 to $7 \%$ of patients with non alcoholic fatty liver disease (NAFLD) [5]. CD screening is indicated for NAFLD associated with unexplained anemia, nutritional deficiency, or repeated abdominal pain. It should be noted that there is a risk of NAFLD after follow-up of the GFD, it is therefore necessary to ensure a strict monitoring of the weight after follow-up of the GFD in order to avoid the overweight related to the correction of the intestinal absorption.

\section{Celiac Disease and Virus Hepatitis}

The treatment of $\mathrm{C}$ hepatitis by the old regimen: interferon and ribavirin resulted in reactivation of some latent CD [6]. Currently, this risk is zero with treatment with direct antivirals. Immunization after vaccination against $\mathrm{B}$ hepatitis is reduced during $\mathrm{CD}$ and corrected under GFD.

\section{Advanced Liver Injury During Celiac Disease}

It has been shown that the risk of developing hepatic cirrhosis is multiplied by 2.23 in celiac patients compared to non-celiac patients [6]. CD can be associated with different types of liver disorders so serological screening of $C D$ should be made during the evaluation of patients with cirrhosis. The mortality rate from hepatic cirrhosis is high during CD compared to the general population [7]. In some studies, it has been reported that well followed GFD, in hepatic cirrhotic patients candidates for liver transplantation, allowed improvement of liver function with removal of patients from the waiting list.

\section{Practical Recommendations}

According to the 2019 recommendations of the European Society for the Study of Celiac Disease (ESsCD) [8], screening for $\mathrm{CD}$ is indicated for unexplained hypertrasaminasemia, and liver assessment is recommended for newly diagnosed CD. The elevation of transaminases generally does not exceed five times the upper limit of normal. A significant elevation exceeding five times the upper limit of normal in a celiac patient requires etiological research. In the absence of liver enzyme normalization after a strict year of GFD, histological evaluation of liver injury should be performed [9]. Cryptogenic hypertransaminemia is asymptomatic.
In case of association with signs suggestive of chronic liver disease: jaundice, ascites, splenomegaly, signs of portal hypertension or liver failure, liver investigations should be performed for chronic liver disease associated with CD [9].

\section{Conclusion}

Liver abnormalities in celiac disease are dominated by celiac hepatitis which may be the only sign of celiac disease and with responds to the gluten free dite. More specific liver abnormalities may be associated with celiac disease with an uncertain response to the gluten-free diet. Hepatic cirrhosis is more common in the celiac population compared to the general population and the effect of the gluten-free diet seems beneficial.

\section{Acknowledgement}

None.

\section{Conflict of Interest}

No conflict of interest

\section{References}

1) Mohammad Rostami-Nejad, Thea Haldane, David Aldulaimi, Seyed Moayed Alavian, Mohammad Reza Zali, et al. (2013) The role of celiac disease in severity of liver disorders and effect of a gluten free diet on diseases improvement. Hepat Mon 13(10): e11893.

2) Linnea Äärelä, Samuli Nurminen, Laura Kivelä, Heini Huhtala, Markku Mäki, et al. (2016) Prevalence and associated factors of abnormal liver values in children with celiac disease. Dig Liver Dis 48(9): 1023-1029.

3) G Novacek, W Miehsler, F Wrba, P Ferenci, E Penner, et al. (1999) Prevalence and clinical importance of hypertransaminasaemia in coeliac disease. Eur J Gastroenterol Hepatol 11(3): 283-288.

4) Lawson A, West J, Aithal GP, RFA Logan, et al. (2005) Autoimmune cholestatic liver disease in people with coeliac disease: a populationbased study of their association. Aliment Pharmacol Ther 21(4): 401405.

5) MT Bardella, L Valenti, C Pagliari, M Peracchi, M Farè, et al. (2004) Searching for coeliac disease in patients with non-alcoholic fatty liver disease. Dig Liver Dis 36(5): 333-336.

6) Rubio-Tapia A, Murray JA (2007) The liver in celiac disease. Hepatology 46(5): 1650-1658.

7) Holmes GKT, Muirhead A (2018) Mortality in coeliac disease: a population-based cohort study from a single centre in Southern Derbyshire, UK. BMJ Open Gastroenterol 5(1): e000201.

8) Abdulbaqi Al-Toma, Umberto Volta, Renata Auricchio, Gemma Castillejo, David S Sanders, et al. (2019) European Society for the Study of Coeliac Disease (ESsCD) guideline for coeliac disease and other gluten-related disorders. United European Gastroenterology J 7(5): 583-613.

9) Alberto Rubio-Tapia, Joseph A Murray (2007) The Liver and Celiac Disease. Hepatology 46(5): 1650-1658. 